\title{
Finite-time boundary stabilization of fractional reaction-diffusion systems
}

\author{
Run-Jie Zhang ${ }^{1}$, Liming Wang ${ }^{1}$, and Kai-Ning $\mathrm{Wu}^{2}$ \\ ${ }^{1}$ Harbin Institute of Technology Weihai \\ ${ }^{2}$ Harbin Institute of Technology - Weihai
}

January 8, 2022

\begin{abstract}
This paper investigates the boundary finite-time stabilization of fractional reaction-diffusion systems (FRDSs). First, a distributed controller is designed, and sufficient conditions are obtained to ensure the finite-time stability (FTS) of FRDSs under the designed controller. Then, a boundary controller is presented to achieve the FTS. By virtue of Lyapunov functional method and inequality techniques, sufficient conditions are presented to ensure the FTS of FRDSs via the designed boundary controller. The effect of diffusion term of FRDSs on the FTS is also investigated. Both Neumann and mixed boundary conditions are considered. Moreover, the robust finite-time stabilization of uncertain FRDSs is studied when there are uncertainties in the system's coefficients. Under the designed boundary controller, sufficient conditions are presented to guarantee the robust FTS of uncertain FRDSs. Finally, numerical examples are presented to verify the effectiveness of our theoretical results.
\end{abstract}

\section{Hosted file}

ZRJ-MMA.pdf available at https://authorea.com/users/454468/articles/552081-finite-timeboundary-stabilization-of-fractional-reaction-diffusion-systems

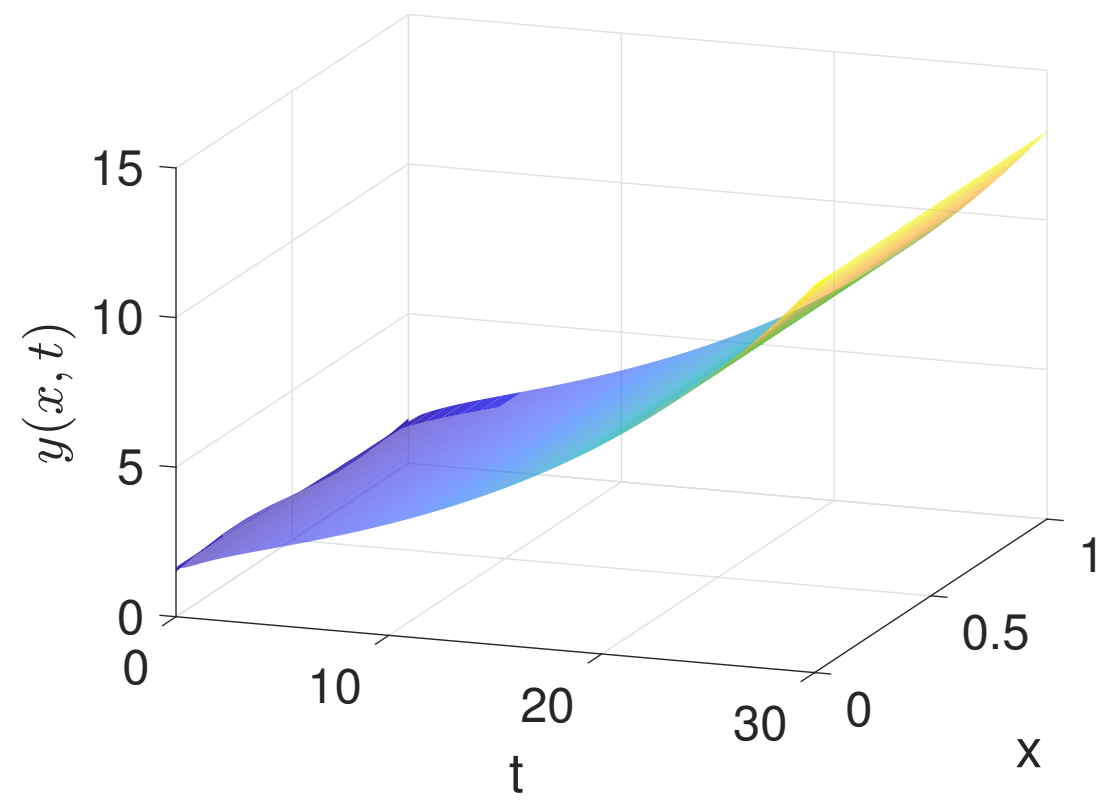



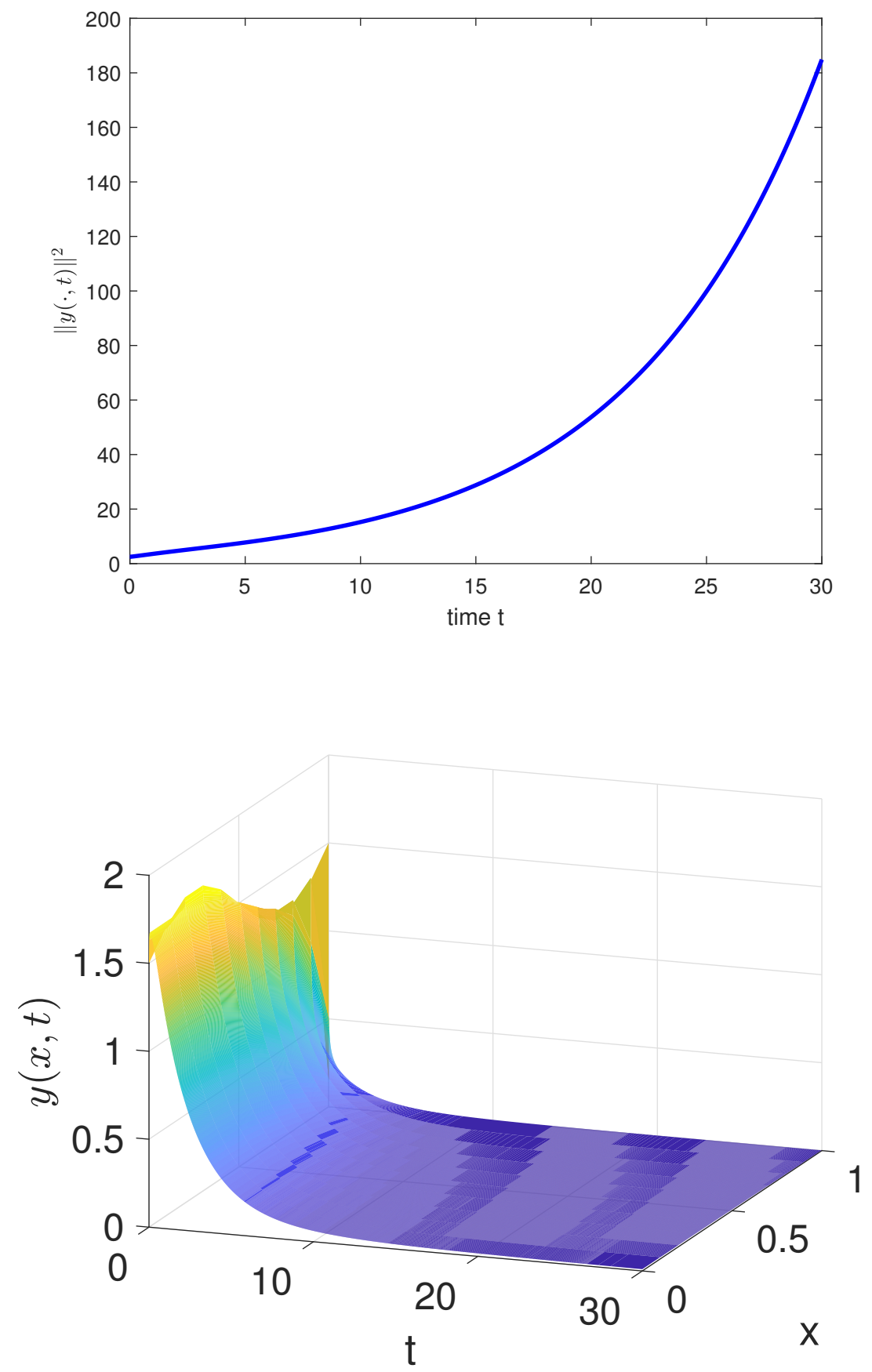

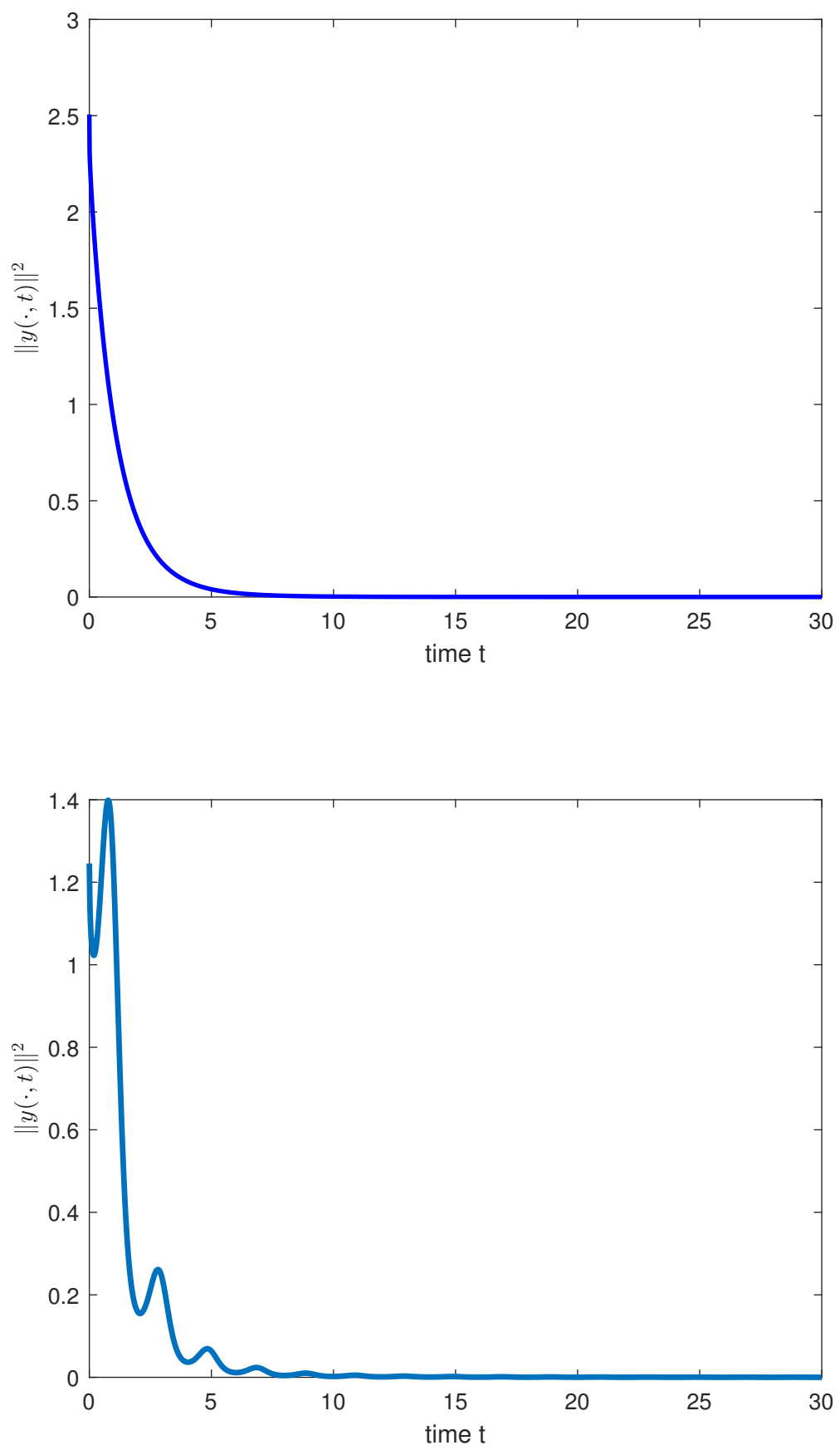

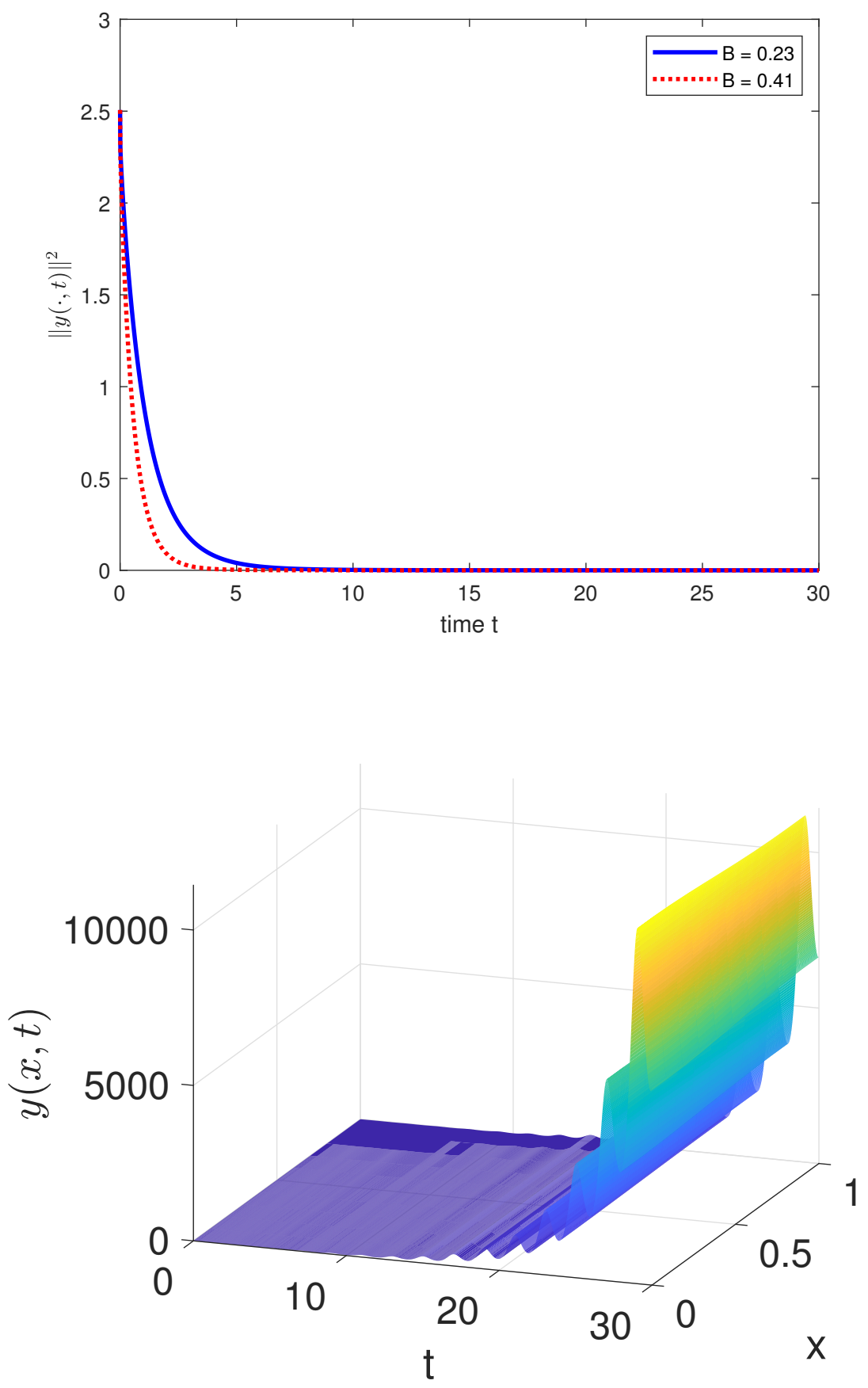

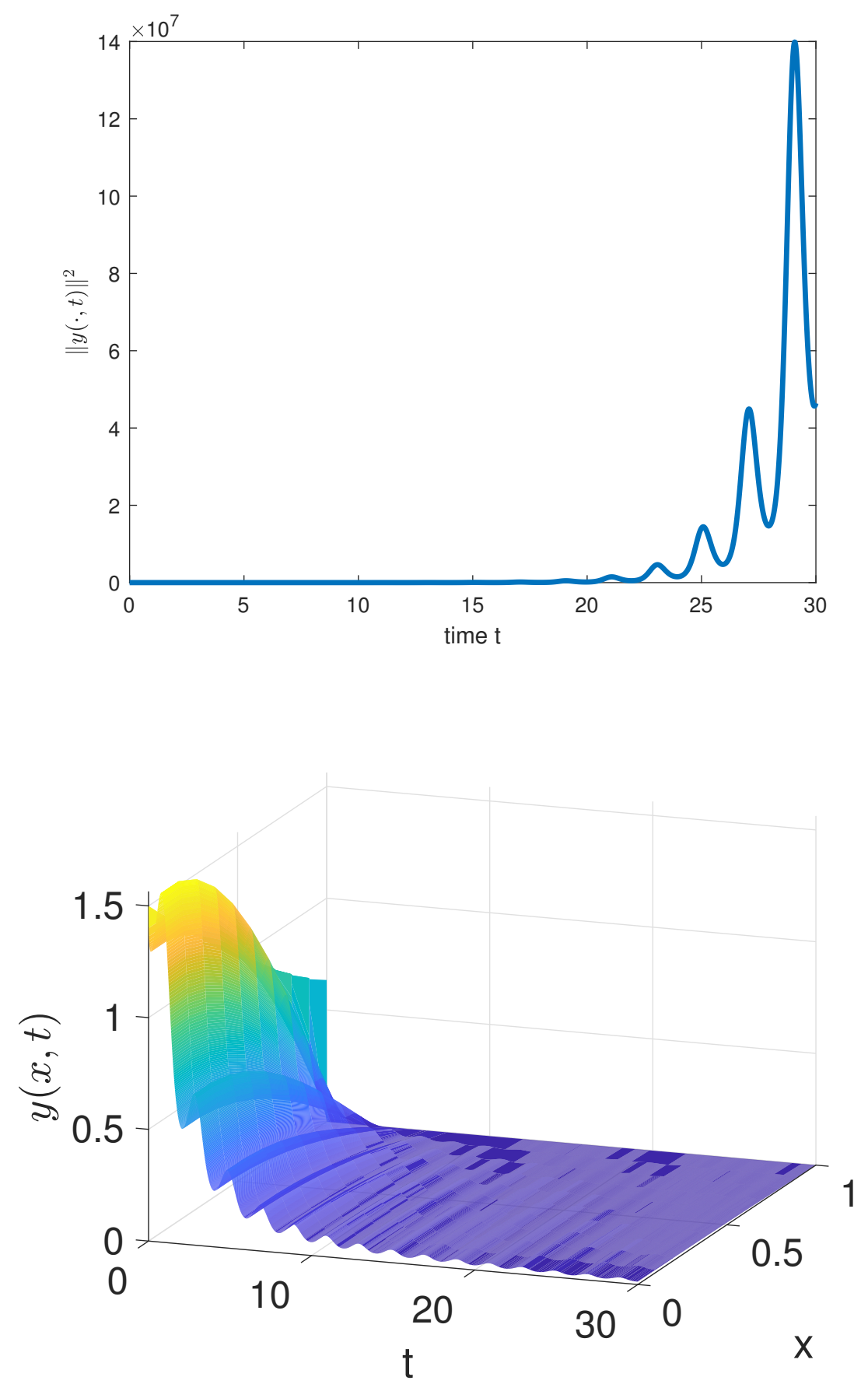